\title{
Autorinnen und Autoren
}

Josi Rom, geb. 1954, Dr. med., Psychiater, Psychoanalytiker, Psychotherapeut und Supervisor in eigener Praxis in Winterthur. Lehrtätigkeit am PSZ und Gründungsmitglied des Dachverbandes Deutschsprachiger PsychosenPsychotherapie (DDPP). Seit 2007 Dozent am Psychosesymposium der Charité Berlin. Mit seiner Publikation «Identitätsgrenzen des Ich - Einblicke in die innere Welt schizophrenie- und borderlinekranker Menschen» ist er 2007 an eine breitere Öffentlichkeit getreten.

Julia Braun, geb. 1969, Dipl.-Psych., Psychoanalytikerin in psychiatrischpsychotherapeutischer Praxis in Zürich. Teilnehmerin am PSZ. Gründungsmitglied des Dachverbandes Deutschsprachiger PsychosenPsychotherapie (DDPP). Bis 2011 leitende Psychologin in einem Rehabilitationszentrum für junge Erwachsene mit Schizophrenie oder Persönlichkeitsstörung. Supervision bei Josi Rom.

Maribel Fischer, geb. 1961, Dr. phil., Psychologin für Psychotherapie FSP, Psychoanalytikerin in eigener Praxis in Winterthur, Mitglied und Dozentin am PSZ.

Christian Scharfetter, geb. 1936, Dr. med., Prof. extraord. der Universität Zürich, emeritus. Publikationen s. www.scharfetter.ch

Dagmar Ambass, geb. 1959, Ethnologin und Psychoanalytikerin, psychotherapeutische Praxis in Zürich und Winterthur. Bis 2010 Familienbegleiterin beim Verein für psychoanalytische Sozialarbeit (vpsz), seitdem Erziehungsberaterin an der Kleinkindberatungsstelle Horgen. Mitarbeiterin im Projekt «Die Fadenspule ein Begegnungsort für kleine Kinder und ihre Eltern». Zurzeit Vorstandsmitglied des Lacan Seminar Zürich.

Stijn Vanheule, geb. 1974, Prof. Dr., ist klinischer Psychologe, Psychoanalytiker in eigener Praxis (Mitglied der holländischsprachigen Kring voor Psychoanalyse der New Lacanian School), und Extraordinarius an der Abteilung für Psychoanalyse und Klinische Beratung der Universität von Gent (Belgien). Seine Forschungsbereiche umfassen lacansche und freudsche Psychoanalyse, psychoanalytische Studien zur Psychopathologie, psychoanalytische klinische Psychodiagnostik, sowie die Methodologie der qualitativen Forschung und der Forschung an kleinen Stichproben. Er ist der Verfasser mehrerer Aufsätze zu diesen Interessensgebieten. 
Markus Brunner, Jan Lohl, Rolf Pohl, Marc Schwietring, Sebastian Winter $(\mathrm{Hg}$. Politische Psychologie heute?

Themen, Theorien und Perspektiven der psychoanalytischen Sozialforschung

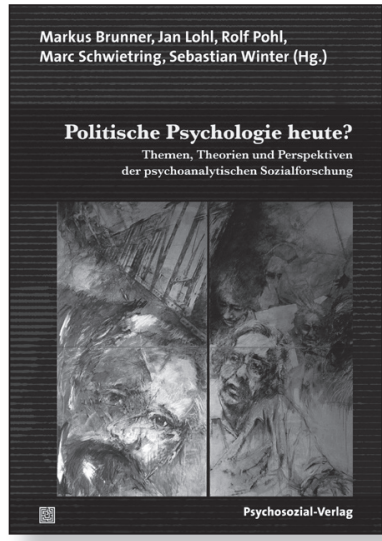

ca. 400 Seiten $\cdot$ Broschur $€ € 36,90$ ISBN 978-3-8379-2118-2

Dieser Band dokumentiert die Tagung »Politische Psychologie heute?«. Diskutiert werden die Bedeutung der politisch-psychologischen Traditionen für die Gegenwart sowie die Möglichkeiten die Politische Psychologie voranzutreiben. Ein Plädoyer für eine kritische Wissenschaft!
Gerald Mackenthun

Gemeinschaftsgefühl

Wertpsychologie und Lebensphilosophie seit Alfred Adler

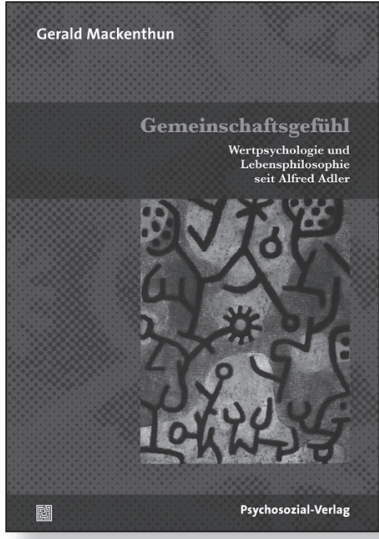

525 Seiten $\cdot$ Broschur $€$ 49,90 ISBN 978-3-8379-2148-9

Die von Alfred Adler 1911 begründete Individualpsychologie basiert auf dem Begriff des Gemeinschaftsgefühls. Im vorliegenden Buch stellt der Autor Adlers Ansatz in seiner historischen Entfaltung vor und zeichnet die engagierte Kontroverse über dieses Konzept innerhalb und außerhalb der Individualpsychologie detailliert nach. 


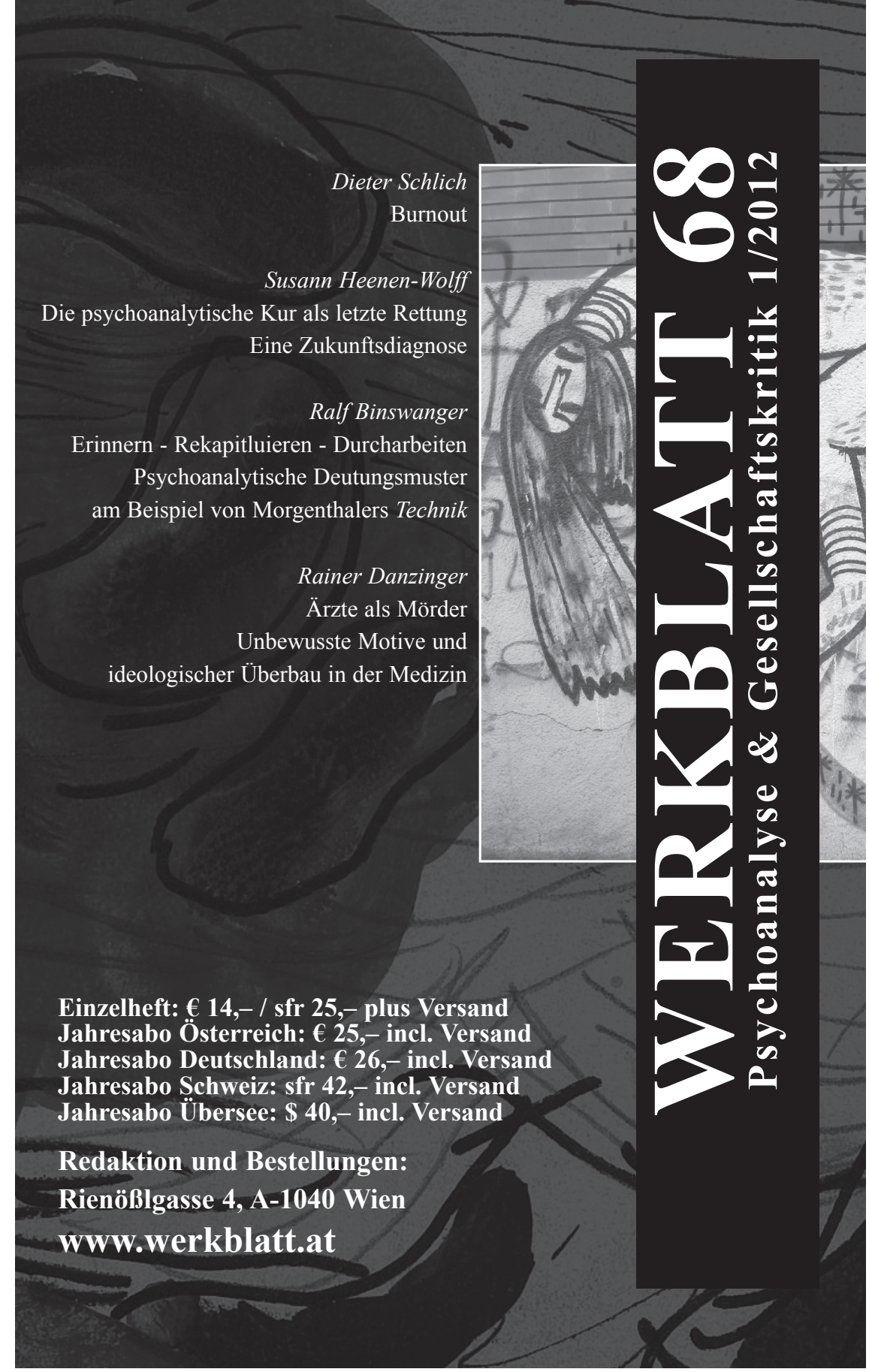


